\title{
Theoretical Study of the Ferroelastic Domain Structure in $\mathrm{La}_{0.95} \mathrm{Sr}_{0.05} \mathrm{Ga}_{0.9} \mathrm{Mg}_{0.1} \mathrm{O}_{3-x}$
}

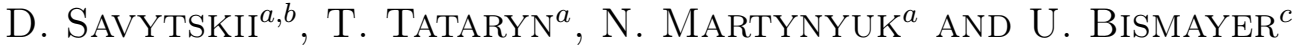 \\ ${ }^{a}$ Lviv Polytechnic National University, 12 Bandera St., 79013 Lviv, Ukraine \\ ${ }^{b}$ SRC "Carat", 202 Stryjska St., 79031 Lviv, Ukraine \\ ${ }^{c}$ Universität Hamburg, Grindelallee 48, D-20146 Hamburg, Germany
}

\begin{abstract}
Theoretical analysis of the ferroelastic domain structure of a $\mathrm{La}_{0.95} \mathrm{Sr}_{0.05} \mathrm{Ga}_{0.9} \mathrm{Mg}_{0.1} \mathrm{O}_{2.925}$ crystal in three different crystallographic phases is presented. Parameters of these configurations are obtained using group theoretical approach, the method of spontaneous deformation as well as theoretical interpretation of twinning resulting from mechanical deformation (mechanical twinning theory). In the three phases of $\mathrm{La}_{0.95} \mathrm{Sr}_{0.05} \mathrm{Ga}_{0.9} \mathrm{Mg}_{0.1} \mathrm{O}_{2.95}-$ trigonal, orthorhombic and monoclinic - the parameters of ferroelastic domain structures are determined; namely the quantity of orientation states, symmetry elements of connection between states, orientations and types of domain walls, tensors of spontaneous deformations of the perovskite-type cells for every orientation state, elements of twin shifts, which are needed for the reorientation of some orientation states to others. By using the found parameters of bidomain configurations a mechanism is proposed, which causes chevron-like domain configurations in compounds with martensitic phase transitions.
\end{abstract}

PACS numbers: $61.72 . \mathrm{Mm}, 61.72 .-\mathrm{y}, 61.50 . \mathrm{Ah}$

\section{Introduction}

There is a great demand for high-efficiency and environmentally appropriate energy sources. Fuel cells belong to such sources. The field of their possible application includes power installations, ranging from power supplies of portable electronic devices to power plants, which can operate in the range from $1 \mathrm{~W}$ up to $10 \mathrm{MW}$ [1-3]. Today, solid oxide fuel cells (SOFC) seem to be the most promising energy sources $[1,4-6]$. Though solid oxide fuel cells have shown many advantages, the SOFC-technology has serious difficulties to develop the optimum fuel and inexpensive alternative materials and technologies.

Recently, lanthanum gallate doped with strontium and magnesium has shown great potential for solid-state oxide fuel cells $[1,4,5,7-10]$. This electrolyte material possesses an ion conductivity exceeding that of zirconium oxide stabilized by yttrium oxide. Other perovskite-type compounds with mixed conduction can be used in SOFC as electrodes and coupling elements. In order to control and improve the critical parameters of these compounds such as the ionic and electron conductivity or thermal expansion, it is necessary to know the dependence of these parameters upon chemical composition as well as on defects and domain structures of perovskites. Hence, it is necessary to carry out detailed studies in order to clarify the real structure of these materials.

Twin domains and their walls are dominant structural features of perovskite-type materials. It has been proved theoretically and experimentally that the diffusion of ionic impurities or vacancies along the twin walls is faster than in the bulk $[11,12]$. Impurities can also be localized in a domain wall or close to it [13-15]. Different transport properties of domain walls and bulk materials can provide the explanation for high ionic conductivity of heavy-twinned perovskites and lanthanum galate doped with strontium and magnesium in particular $[16,17]$.

Previous attempts to understand the influence of the domain (twin) structure on the physical properties were focused on computer simulation because the atomic size of twin walls made it difficult to get reliable experimental data. Most publications about experimental studies of domain walls deal with the direct visualization of domains using transmission electron microscopy [14, 15, 18-23]. At the same time "white" synchrotron radiation re-actualizes the classical Laue method: now it is possible to record thousands of diffraction spots from crystalline samples in a very short time using a charge-coupled device $(\mathrm{CCD})$ area detector.

To avoid identification mistakes one needs to perform complex experimental and theoretical investigations of ferroelastic domain structures. There are often a few orientation states observed with complicated configurations of domain walls. Three different theoretical approaches supplementing each other were used to analyze the parameters of bidomain configuration of the $\mathrm{La}_{0.95} \mathrm{Sr}_{0.05} \mathrm{Ga}_{0.9} \mathrm{Mg}_{0.1} \mathrm{O}_{2.95}$ (LSGM05) twin structure. For calculating the stress-free domain wall orientation, we used a method based on deformation tensors, which describe the spontaneous strains in neighboring domains $[24,25]$. Energy and symmetry aspects of the formation of domain structure are in direct relation because 
the minimum of free energy of the crystal defines the symmetry of this structure. Janovec showed [26] that the number and symmetry elements of domains can be determined via group theory analysis of changes in the point and space symmetry of a crystal during a ferroelastic phase transition. Theoretical description of the twin structure by presenting the twinning as a mechanical deformation has a long history and the underlying principle is well known [27, 28]. Further development and systematization were given in [29-31]. Since this approach essentially complements the previous two ones and allows to determine the direction of shifts necessarily involved in the domain re-orientation, we have considered the LSGM05 ferroelastic domain structure in terms of mechanical twinning. For the determination of multidomain configurations in the studied phases, a method based on transformation matrices was used [32].

The aim of this work is to determine all possible patterns and peculiarities of bidomain as well as polydomain configurations of domain structures in the three distinguished ferroelastic phases of $\mathrm{La}_{0.95} \mathrm{Sr}_{0.05} \mathrm{Ga}_{0.9} \mathrm{Mg}_{0.1} \mathrm{O}_{2.925}$.

\section{Background of theoretical approach}

Crystallographic studies of the $\mathrm{ABO}_{3}$ perovskite oxide family show that only a few of them have the ideal cubic cell at room temperature. Most perovskite cells are distorted because the sizes of $\mathrm{A}$ and $\mathrm{B}$ ions do not fit the structural cavities, which they fill (cube-octahedral and octahedral). Therefore anion cube octahedra or octahedra are distorted or rotated in order to ensure the maximum number of cation-anion contacts. Such structure contains perovskite pseudo-cells with symmetry below cubic, which originates from cubic through small deformations.

In 1960, Indenbom firstly discovered phase transitions, which lead to the spontaneous deformation. Then Aizu introduced the special term "ferroelastic" in solid state physics and explained that ferroelastic phase transitions can be considered separately [33, 34]. During the phase transition from a paraelastic phase (which is the ideal cubic one for perovskites) into the ferroelastic one (characterized by the spontaneous deformation of the ideal perovskite cell) in the mechanically free state, i.e. when there is no external mechanical stresses, the ferroelastics form domains to ensure the total/sum deformation of a sample to be zero. Domains (twins) are regions with constant spontaneous deformation. Roitburd hypothesized [35] that internal stresses appearing in the crystal at the phase transition can extend to rather long distances and play an essential role in ferroelastics. Such stresses considerably decrease in the ferroelastics phase when the crystal is split into two or more domain states with inherent deformation. Hence, when long range elastic stresses relax, the domain separation is energetically most efficient [35].

\subsection{The study of domain structure in terms of the group theory}

The spontaneous deformation matrix describing an orientation state is a second rank symmetric tensor possessing all symmetry elements of the ferroelastic point group. Aizu [33, 34] and Janovec [26] showed that generally during a ferroelastic phase transition a highly symmetric homogeneous crystal transforms to a heterogeneous system of homogeneous regions with lower symmetry, which create a certain spatial configuration. Symmetry operations resulting in the superposition of one orientation (twin) domain with another one are elements of the point group of the paraelastic phase, which are lost during the transition. Macroscopic physical properties of crystals, in particular the mechanical deformation are translationally invariant and fully described by the symmetry of the crystal point group. The comparison of point groups (their symmetry elements) of the $G$ paraelastic and $F$ ferroelastic phases allows to determine the number of possible domain states, symmetry elements, which can connect those states, and the orientation of several possible domain walls.

For example, if symmetry elements belonging to both the group $G$ and the subgroup $F$ act on the spontaneous strain tensor corresponding to the $D_{1}$ orientation state, then the same $D_{1}$ orientation state will be derived. If the tensor of the $D_{1}$ orientation state is affected by symmetry elements belonging to the group $G$ but not to the subgroup $F$, then the result will be a tensor corresponding to another orientation state $D_{i}$ different from $D_{1}$. If $F$ is the point group of the orientation state $D_{1}$ and $g_{i}$ is the operation transforming the state $D_{1}$ (spontaneous strain tensor) into the state $D_{j}$ (including $D_{1}$ ), then the set of all operations connecting $D_{1}$ and $D_{j}$ is $g_{i} F$. This means that each state has its corresponding left class in the subgroup $F$, which constitutes the expansion $G$ [26]:

$$
G=H_{1}+H_{2}+\ldots+H_{q}
$$

where $H_{i}=g_{i} F$ and $i=1 \ldots q$. Here, the number $q$ of possible orientation states in the ferroelastic phase is equal to the order of the point group $G$ in the paraelastic phase divided by the order of the subgroup $F$ in the ferroelastic phase

$$
q=n_{G} / n_{F} .
$$

Using the class expansion we can analyze the possible domain walls between orientation states $D_{1}$ and $D_{i}$ and determine the orientation of certain walls between these states. Stress-free (coherent) domain walls can occur between two orientation states, if there are corresponding "connection" elements $g_{j} \in H_{i}$ meeting the condition of orientation states permutation. This means that if $g_{j}$ applied to $D_{1}$ yields $D_{i}$, then the same operation $g_{j}$ upon $D_{i}$ should yield $D_{1}$, which indicates the equivalency of the orientation states $D_{1}$ and $D_{i}$. Thus, the ambivalence condition [26] should hold to ensure that the condition of orientation states permutation is also fulfilled

$$
g_{i}^{2} \in F,
$$

where $F$ is a point group of the ferroelastic phase. If a 
symmetry plane connects domains, then this plane may be a composition plane $W$ between corresponding domains. This is obvious from the fact that the mirror reflection with respect to the same $m$ plane results in the identical transformation of an object into itself (1). The 2 nd order rotation also meets the ambivalence condition. Hence, if there is the diad among the elements connecting orientation states $D_{1}$ and $D_{i}$, then the plane containing this axis may be a domain wall between such orientation states ( $S$-wall). In contrast to $W$-walls the orientation of this wall depends on the spontaneous strain tensor and is not characterized by fixed Miller indices. It should be noted that in centrosymmetrical ferroelastics, the same class contains not only the mirror plane but also the diad, which is perpendicular (in the paraelastic phase) to this plane.

\subsection{Spontaneous strain tensors method}

The group theory method allows for theoretical prediction of the number of possible domain walls, the symmetry elements "connecting" these walls as well as the orientation of $W$-type domain walls. To determine the orientation of $S$-type walls we used Sapriel's method of the spontaneous strain tensors to find out all stress-free walls in ferroelastic phases [25].

As a rule the domain walls appearing in a crystal do not induce additional elastic long-range forces, which would increase its free energy. The condition of the absence of such forces determines the orientation of possible stress-free domain walls. Since neighboring domains (orientation states) have different spontaneous deformation, the domain wall between them will be a plane where the change of an arbitrary vector length through the spontaneous deformation of cells will be the same in both neighboring domain states.

The plane of the paraelastic phase which may become a domain wall between orientation states $D_{k}$ and $D_{m}$ in the ferroic phase should meet the condition of mechanical compatibility: deformations of any infinitesimal vector belonging to the domain wall should be equal in both neighboring, $m$ and $k$, orientation states, i.e. the following condition should be fulfilled:

$$
\left(\mathrm{d} s^{k}\right)^{2}-(\mathrm{d} s)^{2}=\left(\mathrm{d} s^{m}\right)^{2}-(\mathrm{d} s)^{2},
$$

where $\mathrm{d} s$ and $\mathrm{d} s^{m}, \mathrm{~d} s^{k}$ are infinitesimal small vectors in the paraelastic and ferroelastic phases, correspondingly. The difference of their square lengths in the ferroelastic and paraelastic phases is

$$
\left(\mathrm{d} s^{m}\right)^{2}-(\mathrm{d} s)^{2}=2 \sum_{i, j}^{3}{ }_{s}^{m} D_{i j} x_{i} x_{j},
$$

where ${ }_{s}^{m} D_{i j}$ are components of the spontaneous strain tensor of the $D_{m}$ orientation state in the ferroelastic phase, and $x_{i}, x_{j}$ are coordinates in the paraelastic phase. With Eqs. (5) and (4) we derive equations of possible surfaces between orientation states $D_{k}$ and $D_{m}$ :

$$
\sum_{i, j=1}^{3}\left[{ }_{s}^{k} D_{i j}-{ }_{s}^{m} D_{i j}\right] x_{i} x_{j}=0 .
$$

If the condition

$$
\operatorname{det}\left|{ }_{s}^{1} D_{i j}-{ }_{s}^{i} D_{i j}\right|=0
$$

holds, then expressions can be derived for each $D_{k}-D_{m}$ pair according to Eq. (6). Such equations can be expressed as a product of two linear multipliers, which correspond to two planar mutually perpendicular oriented domain walls.

The condition of equal volumes $V$ of the ferroelastic and paraelastic cells can be used to determine a parameter of the paraelastic phase cubic lattice at a specified temperature [36]. The analysis of Eqs. (6) allows us for determination of all possible orientations of domain walls in different ferroelastic phases of our perovskites-type ferroelastics. Since Eqs. (6) are written in the paraelastic phase setting, in our case the derived coefficients at variables $x_{i}$ are crystallographic Miller indices of planes in the cubic perovskite setting.

\subsection{Mechanical twinning theory}

When an oriented external mechanical stress is applied to a ferroelastic crystal, the spontaneous strain in individual domains can change their orientation. Under rather strong stresses the crystal can become a single domain crystal with its total deformation reaching saturation. Vice versa, the influence of a specifically oriented external mechanical stress can cause the shear of particular regions of the monodomain ferroelastic crystal and transform it into a polydomain sample. The theoretical analysis based on the interpretation of twinning as a mechanical deformation is an essential counterpart to the two previous methods of theoretical study of the LSGM05 twin structure, because it allows us to determine the directions of shears resulting in the domain re-orientation.

The relative position of twin (domain) states can be formally described as a result of the uniform deformation through a simple shear [27-31]. The uniform deformation is completely determined by an ellipsoid, in which a sphere is transformed. Let this sphere be uniformly deformed along the twinning (or composition) plane $K_{1}$, which passes through the center $O$, perpendicular to the figure plane (Fig. 1a). Since points in the plane $K_{1}$ are not displaced, the intersection of the sphere by this plane is not influenced by the deformation and should be one of circular cross-sections of the deformation ellipsoid. In previously introduced terms, the twinning plane $K_{1}$ is a domain wall between a pair of orientation domains with the first domain initially existing and the second one being formed through the twin shear from a certain region of the first domain. The second circular cross-section $K_{2}$ is symmetric to the principal ellipsoid axes $a$ and $c$ and passes through the point $A^{\prime}$ where the circle and the ellipse located in the figure plane cross each other. It is obvious that all vectors in this plane do not change their lengths; however they rotate at the $2 \alpha$ angle. The middle axis $b$ of the ellipsoid is perpendicular to the figure, 
equal to the sphere radius and coincides with the intersection of two circular cross-sections. The plane of the figure contains the maximal $c$ and minimal $a$ axes of the ellipsoid and is called the displacement plane $N$. The trace of the intersection of the 2nd circular cross-section $K_{2}$ and the displacement plane $N$ is designated $\eta_{2}$ and called axis of the principal zone [30, 31].

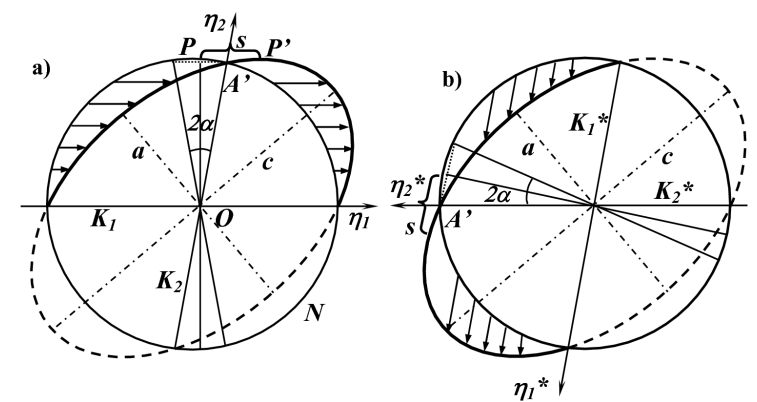

Fig. 1. Reciprocal twinning systems and twinning elements: $K_{1}$ is the composition plane, $\eta_{1}$ is the direction of shear, $K_{2}$ is the second circular section, $\eta_{2}$ is the axis of the principal zone, $N$ is the plane of shear (the plane of the figure), $s$ is the magnitude of shear.

Figure 1b shows that the same ellipsoid can be derived through the twin shear of cell nodes at the same distance $s$ in the direction $\eta_{2}$. In this case, the composition (twinning) plane separating the deformed part (semi-ellipse) and non-deformed part of the crystal (semi-sphere) is the plane $K_{2}$. The second circular cross-section in this new case is $K_{1}$, and $\eta_{1}$ is the axis of the principal zone. Hence, if there is the twin shear with elements $K_{1}, K_{2}, \eta_{1}$ and $\eta_{2}$ in the crystal, then other twin system with twin shear elements $K_{1}^{*}, K_{2}^{*}, \eta_{1}^{*}$ and $\eta_{2}^{*}$ may exist in the centrosymmetric crystals

$$
K_{1}^{*}=K_{2}, \quad K_{2}^{*}=K_{1}, \quad \eta_{1}^{*}=\eta_{2}, \quad \eta_{2}^{*}=\eta_{1} .
$$

Such twin systems are called reciprocal [30]. In the first case, the twin and the non-distorted part are separated by the plane $K_{1}$, in the second case, $K_{1}^{*}=K_{2}$, which is almost perpendicular to the plane $K_{1}$ for the small value $s$ of the crystallographic shear. Twinning elements can be the planes and directions of crystal cell, taking into account the discrete atomic structure of the crystal. Since a twinning shear changes only the cell orientation (one state passes into the other domain state), three possible cases were indicated in respect of rationality or irrationality of the Miller indices of twinning elements [30, 31].

In the case when $K_{1}$ and $\eta_{2}$ have integral Miller indices, a new cell formed by the twin shear is connected with the non-displaced lattice by the mirror reflection through the $K_{1}$ plane. In this case, the twins are called reflection twins or twins of the first kind. If $\eta_{1}$ and $K_{2}$ have integral Miller indices, and $K_{1}$ and $\eta_{2}$ are irrational, then the new lattice is connected with the lattice in the non-displaced region by the $180^{\circ}$ rotation around $\eta_{1}$. These twins are called axial twins or twins of the second kind, because twin states are connected by the $180^{\circ}$ rotation. Very often all four elements of the twin shear $\left(K_{1}, K_{2}, \eta_{1}\right.$ and $\eta_{2}$ ) have integral Miller indices. In this case, both reciprocal twin systems are indistinguishable, and such twin system is called compound twins [30, 31]. In such system, a twin can be considered as a reflection twin or an axial twin at the same time. Using these relations and the results of the group theory analysis, we can determine elements of twin shears in arbitrary ferroelastic phases without special calculations.

\subsection{Multidomain configurations in ferroelastic phases}

For search of allowed variants of multidomain configurations, the method based on transformation matrices, which describe the spacial relationship of two crystal lattice bases for pair of domains with coherent domain wall [32], can be used. Basis vectors $\boldsymbol{a}^{t}, \boldsymbol{b}^{t}, \boldsymbol{c}^{t}$ of an arbitrary domain $D_{t}$ can be expressed through basis vectors $\boldsymbol{a}^{1}, \boldsymbol{b}^{1}, \boldsymbol{c}^{1}$ of the domain $D_{1}$ as following:

$$
\left[\begin{array}{l}
\boldsymbol{a}^{t} \\
\boldsymbol{b}^{t} \\
\boldsymbol{c}^{t}
\end{array}\right]=\boldsymbol{T}_{t 1} \times\left[\begin{array}{l}
\boldsymbol{a}^{1} \\
\boldsymbol{b}^{1} \\
\boldsymbol{c}^{1}
\end{array}\right] \text { or } \boldsymbol{M}_{D t}=\boldsymbol{T}_{t 1} \times \boldsymbol{M}_{D 1}
$$

where $\boldsymbol{T}_{t 1}$ - domain transformation matrix, $\boldsymbol{M}_{D 1}$ orientation matrix of the domain $D_{1}, M_{D t}$ - orientation matrix of the domain $D_{t}$. The above mentioned theoretical group analysis of bidomain ferroelastic (Sect. 2.1) structure allows calculation of transformation matrices $\boldsymbol{T}_{t 1}$ for all domain pairs connected by stress-free coherent domain walls using the derived symmetry elements between domain state pairs. This allows to use the analytical geometry method to determine the spatial orientation of basis vectors $\boldsymbol{a}^{t}, \boldsymbol{b}^{t}, \boldsymbol{c}^{t}$ of an arbitrary domain state $D_{t}$ in Cartesian coordinates with respect to an arbitrary orientation of basis vectors $\boldsymbol{a}^{1}, \boldsymbol{b}^{1}, \boldsymbol{c}^{1}$ of the "reference" domain $D_{1}$ in the same Cartesian coordinates in the case of, for example, reflection twins ( $W$-walls) or axial twinning when domains are related by the rotation at $180^{\circ}$ around the specified direction ( $S$-walls). Then these orientations of basis vectors of both domains in Cartesian coordinates can be used to calculate a transformation matrix $\boldsymbol{T}_{t 1}$ for a corresponding domain pair [32]

It should be noted that a transformation matrix $\boldsymbol{T}_{t 1}$ is calculated to use only lattice parameters of ferroelastic phase. In a result, $\boldsymbol{T}_{t 1}$ offers corresponding symmetry operations of second order directly in ferroelastic phase, i.e. taking into account spontaneous deformations and mutual rotations of domain states, which are peculiar for investigated phase. Besides, the transformation matrices connect orientation matrices, which are used in crystallography for identification and refinement of crystalline structure of crystal by poly- and monochromatic X-ray methods.

There is a stress-free, coherent wall between two orientation states provided that the ambivalence condition (3) is fulfilled. Janovec [31] has formulated this condition in terms of symmetry elements, which are lost during the ferroelastic phase transition. However, it can also be expressed in terms of transformation matrices according to 
$\boldsymbol{T}_{1 t} \times \boldsymbol{T}_{t 1}=\boldsymbol{I}$

where $\boldsymbol{T}_{t 1}=\boldsymbol{T}_{1 t}$ and is the transformation matrix of a domain pair, and $\boldsymbol{I}$ is the unitary matrix [32]. The $\boldsymbol{T}_{t 1}$ transformation matrix transforms the basis vectors of the $D_{1}$ domain into the $D_{t}$ domain vectors. The $\boldsymbol{T}_{1 t}$ transformation matrix holds with respect to the $D_{t}$ basis vectors and we will obtain the $D_{1}$ basis as described by Eq. (10). The $\boldsymbol{T}_{1 t}$ and $\boldsymbol{T}_{t 1}$ transformation matrices are equivalent (equal) just because $D_{1}$ and $D_{t}$ domain states are equivalent [31].

As it was shown in Ref. [32], in order to find other co-existence conditions of more than two domain states without additional stress one may test the criteria (10) for other domain states. For four-domain configuration, the condition of a "stress-free" ferroelastic domain pattern can be written

$$
\boldsymbol{T}_{41} \times \boldsymbol{T}_{34} \times \boldsymbol{T}_{23} \times \boldsymbol{T}_{12}=\boldsymbol{I},
$$

where $\boldsymbol{T}_{12}$ is the transformation matrix related to the domain pair $D_{1}-D_{2}, \boldsymbol{T}_{23}$ - transformation matrix connecting the domain pair $D_{2}-D_{3}, \boldsymbol{T}_{34}$ - transformation matrix is related to the domain pair $D_{3}-D_{4}$, and the $D_{4}-D_{1}$ domain pair is connected by $\boldsymbol{T}_{41}$ transformation matrix. Equation (11) describes the transformation of $D_{1}$ domain basis vectors into $D_{2}$ domain basis vectors by means of the $\boldsymbol{T}_{12}$ transformation matrix followed by three further transformations. They include the $D_{2}-D_{3}$ transformation using $\boldsymbol{T}_{23}$, the $D_{3}-D_{4}$ transformation using $\boldsymbol{T}_{34}$ and return to $D_{1}$ again using the $\boldsymbol{T}_{41}$ transformation matrix. If the result is not the unitary matrix $\boldsymbol{I}$, then the domain configuration would be stressed because the basis vectors of the orientation states cannot be linked without additional strain. It is obvious that Eq. (11) should be satisfied for cyclic permutation of multipliers that is equivalent to the selection of other domain states as a starting point.

\section{Possible domain configurations in ferroelastic phases of LSGM05 crystal}

The study of the LSGM05 crystal structure by the high resolution synchrotron powder diffraction [37] has demonstrated that the orthorhombic symmetry (Imma space group) is characteristic at room temperature. Heating the LSGM05 crystal up to 520-570 K involves phase transition, with the crystal point symmetry being reduced to the monoclinic one ( $I 2 /$ a space group). Further heating to $720 \mathrm{~K}$ results in the transition to the $R 3 c$ non-centrosymmetrical trigonal space group, which transforms in the centrosymmetric one ( $R \overline{3} c$ space group) at approximately $870 \mathrm{~K}$. Our extrapolation of the cell parameters at high temperature shows that the LSGM05 structure is expected to transform into the paraelastic cubic structure near the melting point. Correspondingly, Pm3m space group symmetry describes the paraelastic phase and elements of the $m 3 m$ point group symmetry are characteristic for it. The LSGM05 crystal structure can be considered as a small deformation of an ideal cubic (paraelastic) perovskite cell with the $G=m 3 m$ point group symmetry. The deformations produce the crystal structures of the $\overline{3} m$ and $3 m$ point group symmetry in the trigonal phase, the $2 / m$ point group symmetry in the monoclinic phase, and the $\mathrm{mmm}$ point group symmetry in the orthorhombic one.

The order of the $G$ point group is $n_{\mathrm{G}}=48$. In LSGM05 crystals, there may be 4 orientation states in the centrosymmetrical trigonal phase, 8 states - in the non-centrosymmetrical phase, 12 - in the monoclinic phase, and 6 - in the orthorhombic one. The results of the theoretical analysis for all revealed LSGM05 phases are given in Tables I-III.

\section{1. ferroelastic domain structure of LSGM05 in the trigonal phase}

At the phase transition from the cubic to the trigonal phase the angles of the ideal perovskite cells smoothly change from $90^{\circ}$, and elements of both point and translation symmetries are lost in result. Aizu [33] has introduced the denotation $m 3 m F \overline{3} m$ to describe this phase transition from the paraelastic phase into the ferroelastic trigonal one. The perovskite cell is no longer an elementary one. However, the crystal structure (Fig. 2a) contains perovskite pseudo-cells, which can result from the cubic one through the deformation of right angles. Figure $2 \mathrm{~b}$ shows 12 symmetry elements of the point group $\overline{3} m$, which are peculiar to this pseudo-cell.

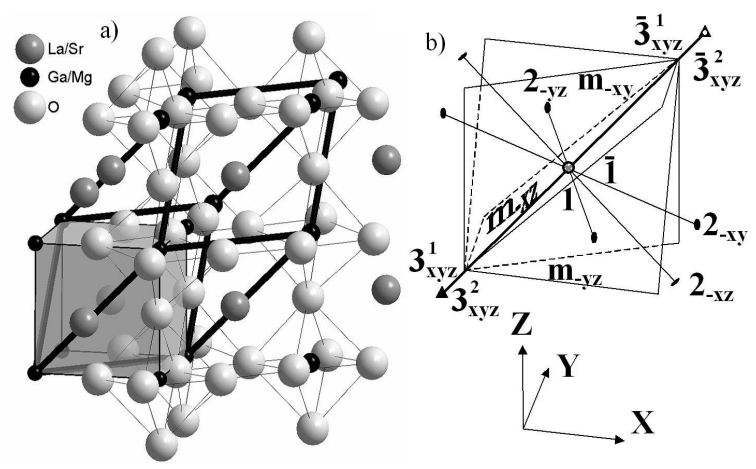

Fig. 2. Model of the LSGM05 crystal structure in the trigonal phase (a) and symmetry elements of point group $\overline{3} m(\mathrm{~b})$.

The relation between the trigonal and the perovskite pseudo-cells can be expressed as following:

$$
\left[\begin{array}{l}
\boldsymbol{a}_{\mathrm{rh}} \\
\boldsymbol{a}_{\mathrm{rh}} \\
\boldsymbol{a}_{\mathrm{rh}}
\end{array}\right]=\left[\begin{array}{lll}
1 & 1 & 0 \\
0 & 1 & 1 \\
1 & 0 & 1
\end{array}\right] \times\left[\begin{array}{l}
\boldsymbol{a}_{\mathrm{p}} \\
\boldsymbol{a}_{\mathrm{p}} \\
\boldsymbol{a}_{\mathrm{p}}
\end{array}\right],
$$

with the perovskite pseudocell $\left(a_{\mathrm{p}}, \alpha_{\mathrm{p}}\right)$ being expressed through the parameters of the trigonal cell $\left(a_{\mathrm{rh}}, \alpha_{\mathrm{rh}}\right)$ according to the following relations:

$$
\begin{aligned}
& a_{\mathrm{p}}=0.5 a_{\mathrm{rh}} \sqrt{3-2 \cos \left(\alpha_{\mathrm{rh}}\right)}, \\
& \alpha_{\mathrm{p}}=\arccos \left(\frac{2 \cos \left(\alpha_{\mathrm{rh}}\right)-1}{3-2 \cos \left(\alpha_{\mathrm{rh}}\right)}\right) .
\end{aligned}
$$




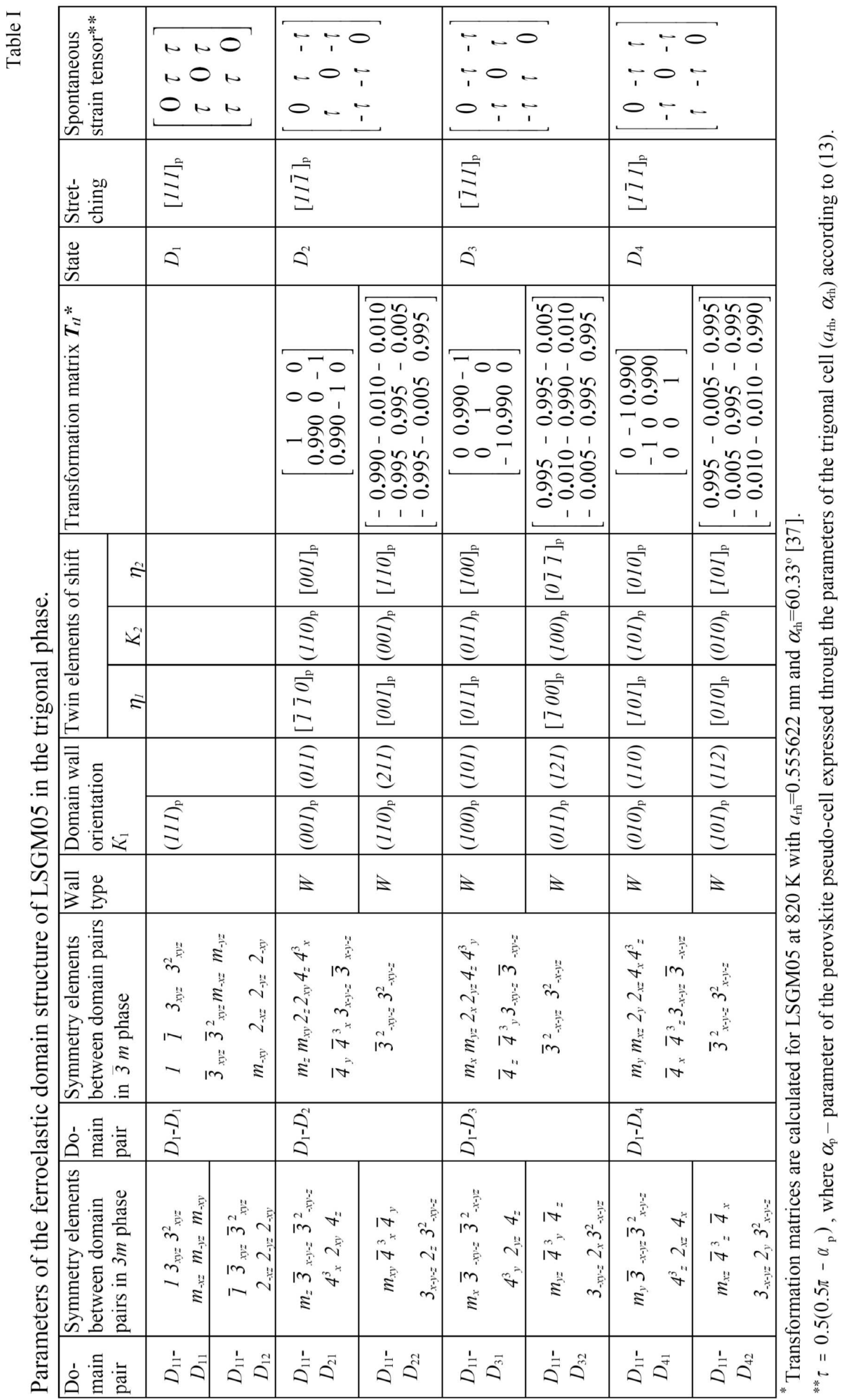




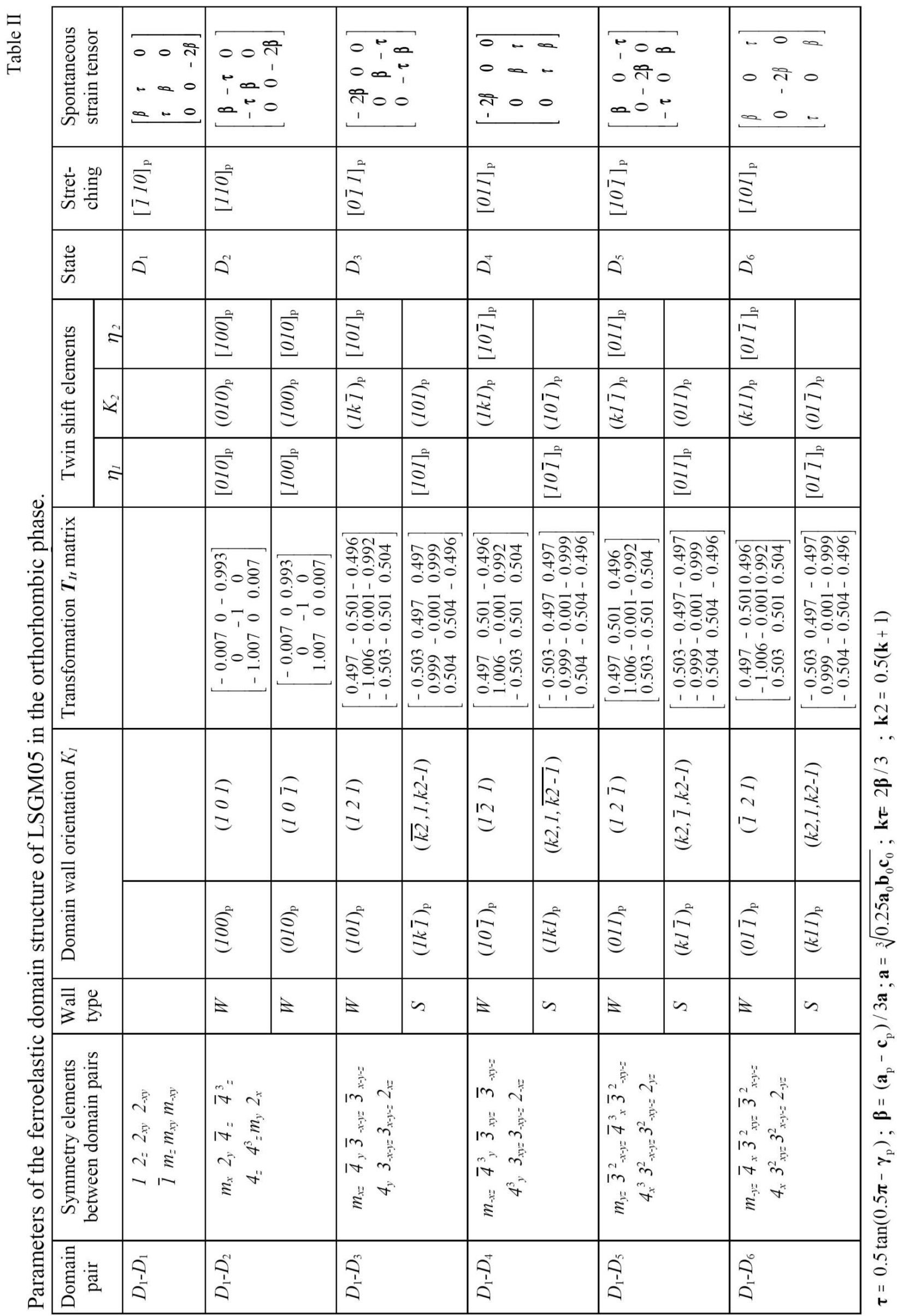


Theoretical Study of the Ferroelastic Domain Structure...

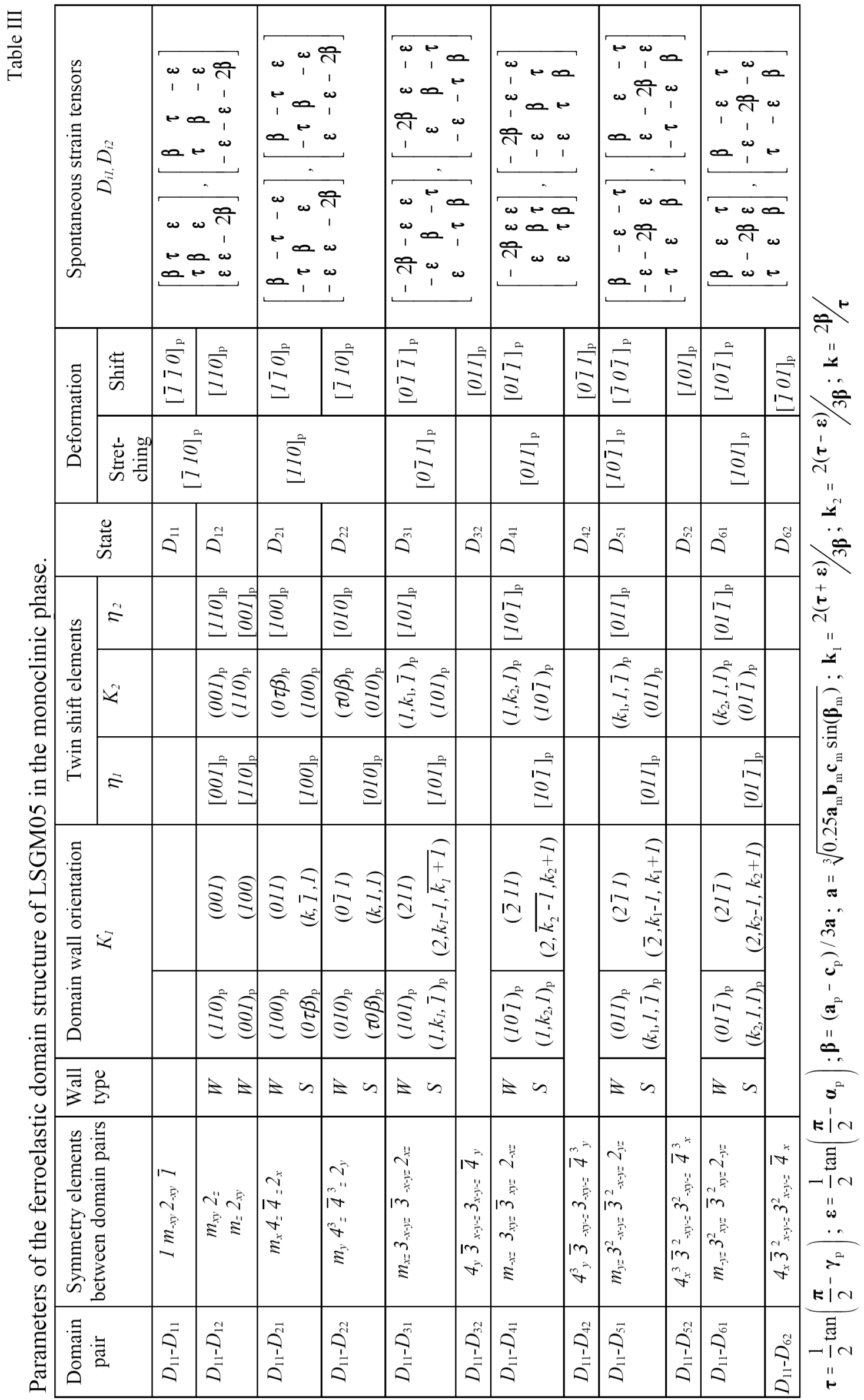


The order of the paraelastic point group $G$ is $n_{\mathrm{G}}=48$. In the centrosymmetrical trigonal phase of LSGM05 crystals, there may be 4 orientation states, in the non-centrosymmetrical phase - 8 states. The results of the group theory analysis for the trigonal phase are given in Table I. We have tested the fulfillment of the conditions (3) and found out that all pairs of orientation states possible in the centrosymmetrical trigonal phase are ambivalent because every corresponding class includes four 2nd order elements (two mirror planes and two diads perpendicular to these planes). According to this, the lateral face $m_{z}$ and the diagonal face $m_{x y}$ of the perovskite cell are the possible domain walls between orientation states $D_{1}$ and $D_{2}$, and the lateral face $m_{x}$ and the diagonal face $m_{y z}$ can connect pair $D_{1}-D_{3}$ whereas the lateral face $m_{y}$ and the diagonal face $m_{x z}$ - pair $D_{1}-D_{4}$. These $W$-walls are crystallographic planes with fixed Miller indices. Their orientations in the perovskite and rhombohedral settings are given in Table I. It should be noticed that if a class includes the mirror plane, then the diad perpendicular (in the paraelastic phase) to this plane belongs to the same class that results from the centrosymmetrical crystal structure.

When the center of symmetry disappears in the trigonal phase, each of four orientation states available in the LSGM05 crystal may be divided into two $180^{\circ}$ domains with inverse dipole moments. The most probable domain walls between these new $180^{\circ}$ domains $D_{11}-D_{12}$ is $(111)_{\mathrm{p}}$ plane containing all three 2 nd order axes $2_{-x z}, 2_{-y z}$ and $2_{-x y}$ (Table I), which are lost during the phase transition from the centrosymmetrical LSGM05 structure to the acentric one (space groups $R \overline{3} c$ and $R 3 c$, correspondingly). Table I shows that 8 domain states appearing in the acentric phase can be neighboring through the same crystallographic planes as 4 orientation states in the centrosymmetrical trigonal phase. Hence the cardinal transformation of the twin structure is unlikely to happen at this phase transition. Hence the Laue class, not a point group, determines the Laue diffraction pattern symmetry, then Laue pattern does not depend upon availability of a center of symmetry in a crystal structure. Therefore, further analysis of the domain structure in the trigonal phase does not take into consideration the separation in $180^{\circ}$ domains in the $720-870 \mathrm{~K}$ temperature range.

The perovskite pseudo-cell in the trigonal phase of the LSGM05 crystal is formed through pressing a cube along the diagonal [111]. However, there are 3 other equivalent

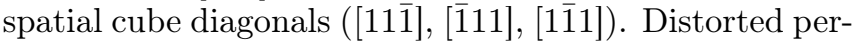
ovskite cells obtained through stretching along these diagonals are similar, but differently oriented in the space. This means that the deformation of the cube through the pressing along spatial diagonals provides 4 possible orientation states (domains) as it was concluded using the group theory. Each of them is described by corresponding spontaneous strain tensor, which is presented in Table I. Using the found tensors, it can be shown that there are no $S$-type domain walls between any pairs of orientation states $D_{1}$ and $D_{i}(i=2,3,4)$ in the trigonal phase, and only $W$-type walls with fixed Miller indices (planes $\{110\}$ and $\{211\}$ in the rhombohedral setting) can be expected in this ferroelastic phase.

According to the group theory analysis, these walls are mirror planes in the paraelastic cubic phase, while in the ferroelastic phase they are symmetry elements connecting the pairs of indicated orientation states (Table I). Furthermore, every pair of domain states is connected by two diads. All these diads lie in the corresponding mirror reflection planes which belong to the same left class (Table I). For example, $\mathscr{2}_{x y}$ is parallel to $m_{z}$ and $2_{z}$ lies in $m_{x y}$. In terms of the mechanical twinning theory this phenomenon is classified as compound twin. In this case, domain states are connected by a mirror plane and a diad at the same time. Therefore the expected $S$-walls should coincide with corresponding $W$-walls. In this case, all four elements of the twin shear $\left(K_{1}, K_{2}\right.$, $\eta_{1}$ and $\eta_{2}$ ) have integer Miller indices and correspond to mirror planes and diads of the same left class (Table I). For example, compound twin with elements $K_{1}(001)_{\mathrm{p}}$, $\eta_{1}[\overline{1} \overline{1} 0]_{\mathrm{p}}, K_{2}(110)_{\mathrm{p}}$ and $\eta_{2}[001]_{\mathrm{p}}$ appears at reorientation of the orientation state $D_{1}$ into the orientation state $D_{2}$ as a result of twin shear in direction $\eta_{1}[\overline{1} \overline{1} 0]_{\mathrm{p}}$. These domains are connected by the mirror reflection relatively plane $K_{1}(001)_{\mathrm{p}}\left(m_{z}\right)$ and the rotation on $180^{\circ}$ around $\eta_{1}[\overline{1} \overline{1} 0]_{\mathrm{p}}\left(2_{x y}\right)$ at the same time (Fig. 3$)$. Another compound twin can appear, for example, at a twin shear of perovskite pseudocell nodes in direction $\eta_{1}[\overline{1} 00]_{\mathrm{p}}$. Then the part of orientation state $D_{1}$ reorients into the orientation state $D_{3}$. In this case, the mirror plane $m_{y z}$ $\left(K_{1}(011)_{\mathrm{p}}\right)$ as well as diad $\mathscr{2}_{x}\left(\eta_{1}\right)$ simultaneously connect basis vectors of domain state $D_{1}$ and $D_{3}$ (Fig. 4 ). This is true for all pairs (two-state configurations) of orientation domains of the trigonal symmetry (Table I).

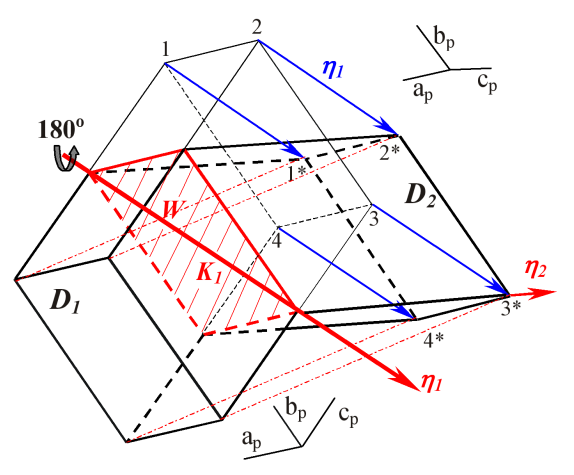

Fig. 3. Compound twin with elements of a twin shear: $K_{1}(001)_{\mathrm{p}}$ (mirror plane), $\eta_{1}[\overline{1} \overline{1} 0]_{\mathrm{p}}$ (axial rotation on $\left.180^{\circ}\right), K_{2}(110)_{\mathrm{p}}, \eta_{2}[001]_{\mathrm{p}}$. Twin shear in the $\eta_{1}$ direction reorients the orientation state $D_{1}$ (perovskites pseudo-cell nodes 1-4) into the orientation state $D_{2}$ (perovskites pseudo-cell nodes $1^{*}-4^{*}$ ) of the trigonal phase.

In real sample, complex multidomain configuration of ferroelastic structure can appear. In Ref. [32] it was shown that the junction of three and five orientation do- 


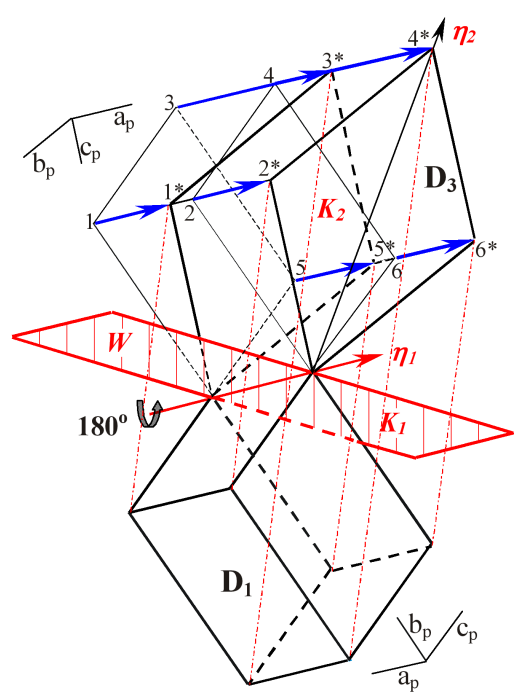

Fig. 4. Compound twin with elements of a twin shear: $K_{1}(011)_{\mathrm{p}}$ (mirror plane), $\eta_{1}[\overline{1} 00]_{\mathrm{p}}$ (axial rotation on $\left.180^{\circ}\right), K_{2}(100)_{\mathrm{p}}, \eta_{2}[0 \overline{1} \overline{1}]_{\mathrm{p}}$. Twin shear in the direction $\mu_{1}$ reorients the orientation state $D_{1}$ (perovskites pseudo-cell nodes 1-6) into the orientation state $D_{3}$ (perovskites pseudo-cell nodes $1^{*}-6^{*}$ ) of the trigonal phase.

mains is strained whereas the encounter of four domains is strain-free, in particular, for examined three different ferroelastic phases in LSGM05. For example, in case of the ferroelastic trigonal phase, 3 different variants were received, when Eq. (11) was fulfilled. All these cases are crystallographically equivalent

$$
\begin{aligned}
& W 110 \times W 121 \times W 011 \times W 121=I, \\
& W 011 \times W 112 \times W 101 \times W 112=I, \\
& W 101 \times W 211 \times W 110 \times W 211=I,
\end{aligned}
$$

where the symbols of domain walls between the relevant orientation states are used instead of the symbols of transformation matrices. Repetition of such 4-domain junctions in a sample volume allows to form a chevron-like domain configuration [32]. Mechanical twinning theory $[30,31]$ implies that a twinning lamella intersects a twinning boundary without strain if the displacement direction $\eta_{1}$ is parallel to the plane of the intersected wall (compositional plane). Our analysis of the "strain-free" twin configurations in LSGM05 shows that this condition holds for the trigonal phase. Thus, in the trigonal structure, the crystal can be divided into two domains with wall (011) (or (001) p using perovskite setting) because certain areas of the crystal shift along direction $[\overline{1} \overline{1} 0]_{\mathrm{p}}$ (Fig. 5a,b). Hence, "chevron-like" configuration or "zig-zag-shaped" lamellae with walls (121) (or (011)p using perovskite setting) appear due to the shift of certain areas along direction $[\overline{100}]_{\mathrm{p}}$ parallel to the previously appearing boundaries (Fig. 5c,d). Other two "chevron-like" domain configurations, which correspond to Eqs. (15) and (16), can be formed in the trigonal phase in a similar way through displacements parallel to the corresponding directions (Table I).

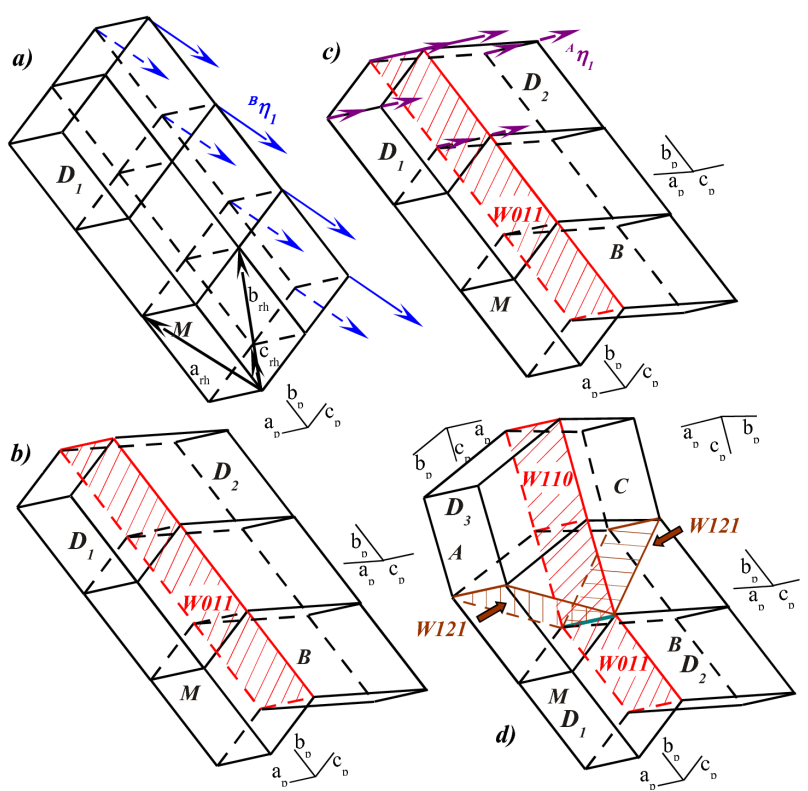

Fig. 5. Sequence of two twinning shears parallel to directions $[\overline{1} \overline{1} 0]_{p}(a)$ and $[\overline{1} 00]_{p}(c)$. From a single domain crystal (a) there originates a two- (b) and a "chevron-like" four-domain pattern (d) in the trigonal ferroelastic phase.

\section{2. ferroelastic domain structure of LSGMO5 in the orthorhombic phase}

At room temperature, LSGM05 has an orthorhombic ferroelastic phase with symmetry elements of the point group $\mathrm{mmm}$. The perovskite monoclinic pseudo-cell has the same point symmetry, $\mathrm{mmm}$ (Fig. 6a,b). The cell parameters can be expressed as

$$
\begin{aligned}
& a_{\mathrm{p}}=b_{\mathrm{p}}=\sqrt{\frac{a_{0}^{2}+c_{0}^{2}}{4}}, \\
& c_{\mathrm{p}}=0.5 b_{0}, \quad \gamma_{\mathrm{p}}=2 \arctan \frac{a_{0}}{c_{0}},
\end{aligned}
$$

and the relation between the cells is

$$
\left[\begin{array}{l}
\boldsymbol{a}_{0} \\
\boldsymbol{b}_{0} \\
\boldsymbol{c}_{0}
\end{array}\right]=\left[\begin{array}{ccc}
1 & 1 & 0 \\
0 & 0 & 2 \\
1 & -1 & 0
\end{array}\right] \times\left[\begin{array}{l}
\boldsymbol{a}_{\mathrm{p}} \\
\boldsymbol{b}_{\mathrm{p}} \\
\boldsymbol{c}_{\mathrm{p}}
\end{array}\right],
$$

where $a_{0}, b_{0}, c_{0}$ are cell parameters of the orthorhombic cell; and $a_{\mathrm{p}}, b_{\mathrm{p}}, c_{\mathrm{p}}$ are cell parameters of the perovskite cell.

The lateral faces $m_{x}$ and $m_{y}$ of the perovskite cell can become domain walls between orientation states $D_{1}$ and $D_{2}$ in the orthorhombic phase (Table II), and $m_{x z}, m_{-x z}$, $m_{y z}, m_{-y z}$ planes (cube mirror planes passing through the pair of opposite cube edges) can connect pairs $D_{1}$ and $D_{3}, D_{1}$ and $D_{4}, D_{1}$ and $D_{5}, D_{1}$ and $D_{6}$. These walls are crystallographic planes with fixed Miller indices. The 2nd order axes $\mathscr{2}_{x}$ and $\mathscr{2}_{y}$ are in the planes $m_{y}$ and $m_{x}$, 


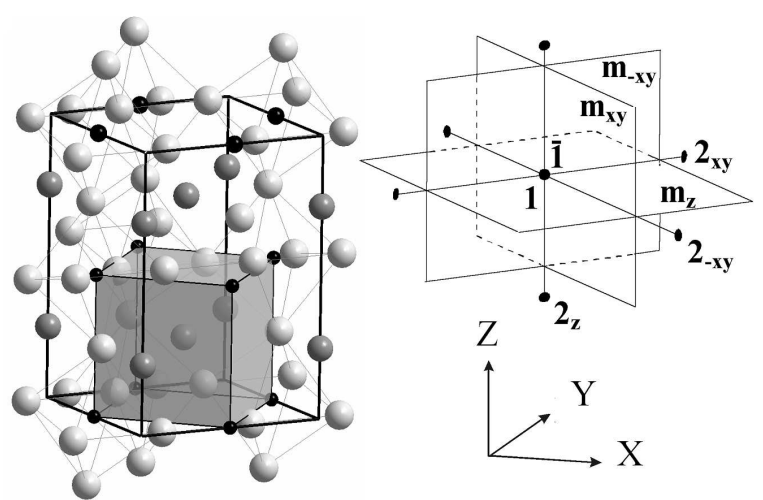

Fig. 6. Model of the LSGM05 crystal structure in the orthorhombic phase (a) and symmetry elements of point group $m m m$ (b).

correspondingly. Thus, similar to the trigonal phase the $S$-walls expected in the orthorhombic phase coincide with their corresponding $W$-walls for $D_{1}$ and $D_{2}$. The states $D_{1}$ and $D_{3}, D_{1}-D_{4}, D_{1}-D_{5}, D_{1}-D_{6}$ may be connected by $S$-walls passing through the lateral diagonals $\left(\mathscr{2}_{x z} ; 2_{-x z}\right.$; $\left.2_{y z} ; \mathscr{2}_{-y z}\right)$ of cube. Their orientation can be determined by the method of spontaneous strain tensors.

The perovskite pseudo-cell of the $\mathrm{La}_{0.95} \mathrm{Sr}_{0.05} \mathrm{Ga}_{0.9} \mathrm{Mg}_{0.1} \mathrm{O}_{2.925}$ orthorhombic phase can be considered as an ideal one stretched along the diagonal $[\overline{1} 10]_{\mathrm{p}}$. There are five more diagonals equivalent to $[1 \overline{1} 0]_{\mathrm{p}}$ in the ideal perovskite cell. The deformation along these diagonals results in equivalent distortions of the cell. Each of the 6 orientation states $D_{i}$ in the orthorhombic ferroelastic phase is characterized by a spontaneous strain tensor with components presented in Table II. Similarly to domain pairs in the trigonal phase, only $W$-type walls may originate between the states $D_{1}$ and $D_{2}$ in the orthorhombic phase (Fig. 7).

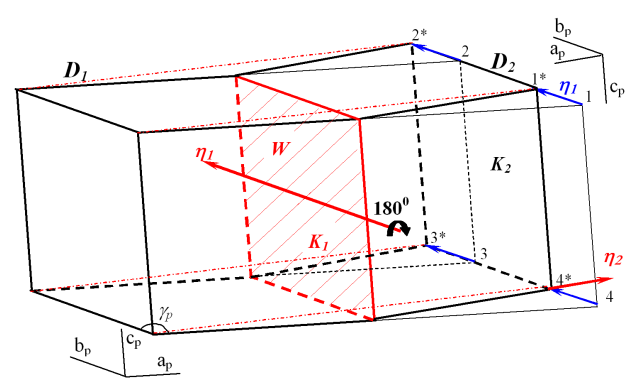

Fig. 7. Compound twin with elements of a twin shear: $K_{1}(100)_{\mathrm{p}}$ (mirror plane), $\eta_{1}[010]_{\mathrm{p}}$ (axial rotation on $\left.180^{\circ}\right), K_{2}(010)_{\mathrm{p}}, \eta_{2}[100]_{\mathrm{p}}$. Twin shear in the direction $\eta_{1}$ reorients the orientation state $D_{1}$ (perovskites pseudo-cell nodes 1-4) into the orientation state $D_{2}$ (perovskites pseudo-cell nodes $1^{*}-4^{*}$ ) of the orthorhombic phase.

In contrast to this domain pair, a $W$-type wall and a perpendicular $S$-wall can connect the domain pairs
$D_{1}-D_{i}(i=3-6)$ in the orthorhombic phase. $\eta_{1}$ and $K_{2}$ have integral Miller indices and $K_{1}$ and $\eta_{2}$ are irrational in those cases when pairs of these orientation states are connected by $S$-type walls. Hence, the "new" twin lattice is connected with the lattice in the non-displaced region through a $180^{\circ}$ rotation around $\eta_{1}$ (Fig. 8). These twins are called "axial twins", because twin states are connected only by $180^{\circ}$ rotation around a 2 nd order axis of the paraelastic cubic phase as follows from group theory. Since at least one of the three Miller indices of $S$-walls are expressed through the tensor components given in Table II, the domain wall rotates around the corresponding axis located in this plane when the temperature changes.

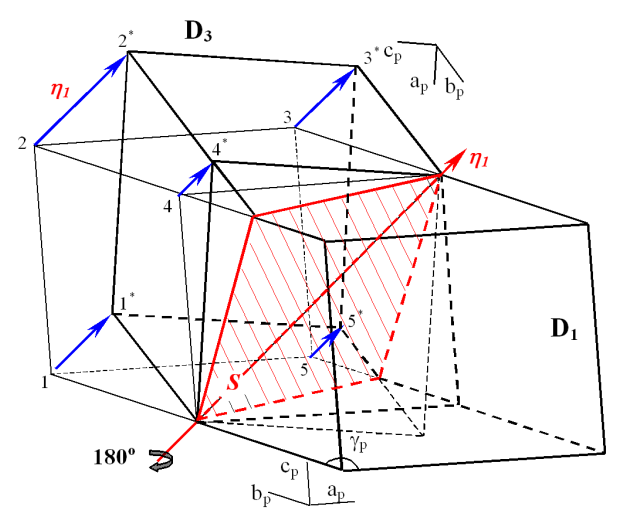

Fig. 8. Axial twin with elements of a twinning shear: $\eta_{1}[101]_{\mathrm{p}}$ and $K_{2}(101)_{\mathrm{p}}$. Twin shear in the direction $\eta_{1}$ reorients the orientation state $D_{1}$ (perovskites pseudo-cell nodes 1-5) into the orientation state $D_{3}$ (perovskites pseudo-cell nodes $1^{*}-5^{*}$ ) of the orthorhombic phase.

It should be noticed that the described cases of axial twinning are reciprocal to twins of the same domain pairs with $W$-type walls (Table II). According to group theoretical analysis, $W$-walls are mirror planes in the paraelastic cubic phase, while in the ferroelastic phase, they are symmetry elements connecting pairs of the indicated orientation states. In those cases, $K_{1}$ and $\eta_{2}$ have integral Miller indices (Table II). As a result of the corresponding twin shear "new" cells of $D_{i}(i=3-6)$ are connected with the non-displaced lattices by mirror reflection through the plane $K_{1}$ (Fig. 9). Such twins are called reflection twins.

In the orthorhombic phase, more variants of strain-free multidomain junctions can appear compared with the trigonal system. Each of these strain-free multidomain junctions consists of four orientation domains [32]. Due to crystallographic equivalence junctions they can be divided into three groups. The first group contains domain walls only of $W$-type. Two other groups contain four-domain junctions with domain walls of $W$ - and $S$-type. Junctions with $W$-type walls $\{101\}$ are attributed to the second group, whereas junctions with $W$-type walls $\{121\}$ belong to the third group. Similarly to the trigonal phase, a repetition (translation) of such 4-domain 


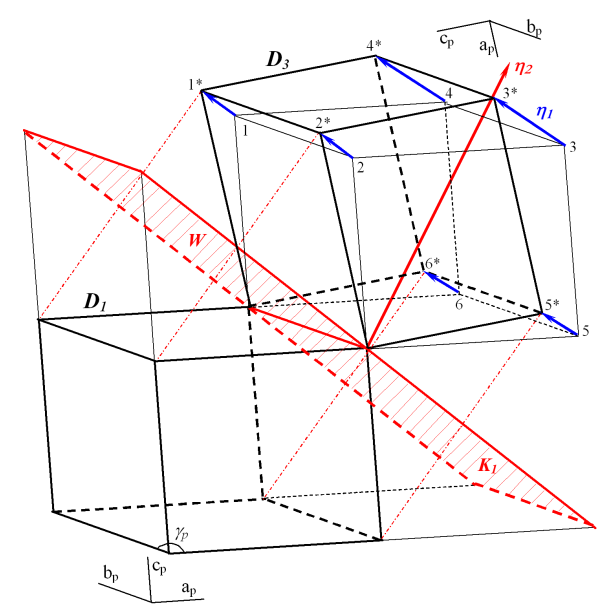

Fig. 9. Reflection twin with elements of a twinning shear: $K_{1}(101)_{\mathrm{p}}$ and $\eta_{2}[101]_{\mathrm{p}}$. Twin shear in the direction $\eta_{1}$ reorients the orientation state $D_{1}$ (perovskites pseudo-cell nodes 1-6) into the orientation state $D_{3}$ (perovskites pseudo-cell nodes $1^{*}-6^{*}$ ) of the orthorhombic phase.

junctions forms chevron-like domain configurations. One of such junctions is shown in Fig. 10, where 4-domain stress-free junctions appear due to two sequential twinning shears. Thus, in the orthorhombic structure, the crystal can be divided into two domains with wall $(12 \overline{1})$ (or $(011)_{\mathrm{p}}$ using perovskite setting) because certain areas of the crystal shift along the direction close to $[021]_{p}$ (Fig. 10a, b). Hence, a "chevron-like" configuration or "zig-zag-shaped" lamellae with walls $(10 \overline{1})$ (or $(010)_{\mathrm{p}}$ using perovskite setting) is formed due to the shift of certain areas along the direction $[100]_{\mathrm{p}}$, parallel to the previously formed boundaries (Fig. 10c,d). Further nine "chevron-like" domain configurations can be formed in the orthorhombic phase in a similar way through two sequential twinning shears.

\section{3. ferroelastic domain structure of LSGM05 in the monoclinic phase}

Similarly to the abovementioned trigonal and orthorhombic structures, the monoclinic phase $\left(a_{m}, b_{m}\right.$, $\left.c_{m}, \beta_{m}\right)$ with point symmetry $2 / m$ is also characterized by the perovskite pseudo-cell. The basic vectors of this cell are related with the vectors of the elementary monoclinic cell

$$
\left[\begin{array}{l}
\boldsymbol{a}_{m} \\
\boldsymbol{b}_{m} \\
\boldsymbol{c}_{m}
\end{array}\right]=\left[\begin{array}{ccc}
0 & 0 & 2 \\
1 & -1 & 0 \\
1 & 1 & 0
\end{array}\right] \times\left[\begin{array}{c}
\boldsymbol{a}_{\mathrm{p}} \\
\boldsymbol{b}_{\mathrm{p}} \\
\boldsymbol{c}_{\mathrm{p}}
\end{array}\right]
$$

where

$$
\begin{aligned}
& a_{\mathrm{p}}=b_{\mathrm{p}}=\sqrt{\frac{b_{m}^{2}+c_{m}^{2}}{4}}, \quad c_{\mathrm{p}}=0.5 a_{m}, \\
& \gamma_{\mathrm{p}}=2 \arctan \frac{b_{m}}{c_{m}},
\end{aligned}
$$

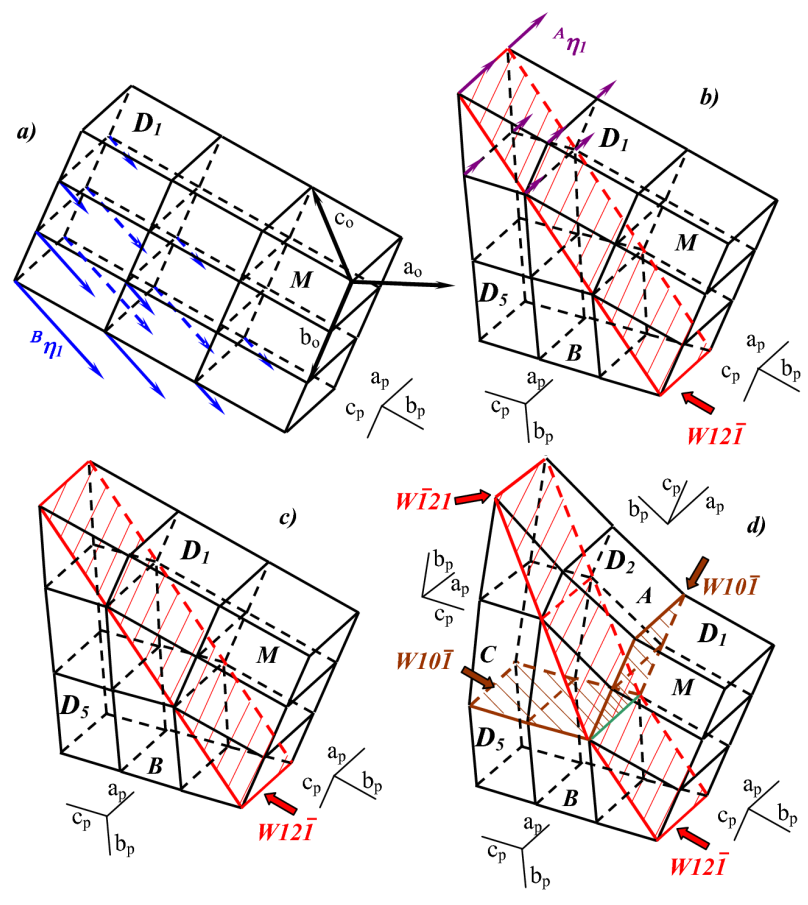

Fig. 10. Sequence of two twinning shears parallel to directions $[021]_{\mathrm{p}}(\mathrm{a})$ and $[100]_{\mathrm{p}}(\mathrm{c})$. From a single domain crystal (a) there originate two- (b) and "chevron-like" four-domain patterns $(d)$ in the orthorhombic ferroelastic phase.

$\alpha_{\mathrm{p}}=\beta_{\mathrm{p}}=\arccos \left(\cos \left(\beta_{m}\right) \cos \left(0.5 \gamma_{\mathrm{p}}\right)\right)$.

The perovskite pseudo-cell of the monoclinic phase is formed from the ideal perovskite cell through stretching along $[110]_{\mathrm{p}}$ like in the orthorhombic phase, followed by a simple spatial shear in the perpendicular direction. In the case of shear along $[\overline{1} \overline{1} 0]_{\mathrm{p}}$, the orientation state $D_{11}$ appears, and for a shear along direction $[110]_{\mathrm{p}}$ the orientation state $D_{12}$ appears (Fig. 11). The other 10 orientation states of the monoclinic phase are formed through stretching of the cell along five other cubic diagonals, which are crystallographically equivalent to $[\overline{1} 10]_{\mathrm{p}}$, plus simple uniform shears in two opposite directions perpendicular to the corresponding diagonal. Spontaneous strain tensors of the perovskite pseudo-cells are given in Table III.

Consequently, each of six orientation states available in the orthorhombic phase of LSGM05 crystal can be divided into two domains (Fig. 11, Table III) during the phase transition from the orthorhombic to the monoclinic phase. In this case, all domain walls of both, $W$ and $S$ types, allowed in the orthorhombic phase are also likely to form in the monoclinic ferroelastic phase. Additionally, walls with the other orientations can also appear, like $W$-walls $m_{x y}, m_{z}$ and $S$-walls containing the 2 nd order axes $\mathscr{2}_{x}$ and $\mathscr{2}_{y}$ (Table III). It should be noticed that some domain states, for example, $D_{11}$ and $D_{32}, D_{11}$ and $D_{42}$, $D_{11}$ and $D_{52}, D_{11}$ and $D_{62}$ pairs cannot be adjacent, because the ambivalence condition (3) is not fulfilled for 
the connecting symmetry elements. Therefore, domain states cannot match each other through a planar surface without stresses. Such domain states can coexist in the same sample provided they are separated by one more domain state which is neighboring with the states above through stress-free domain walls.

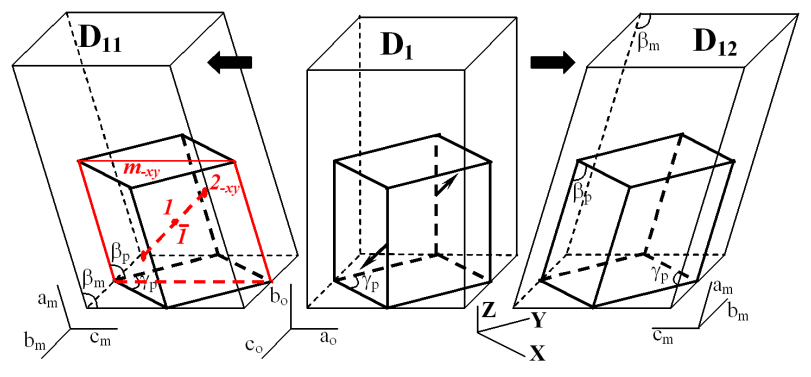

Fig. 11. Relationship between basic vectors of the perovskite pseudo-cell and the elementary cell for the domain state $D_{1}$ of the orthorhombic phase and domain states $D_{11}$ and $D_{12}$ of the monoclinic phase. Relevant deformations of the perovskite pseudo-cells and their symmetry elements of point group $(2 / m)$ are marked.

As one can see in Table III, there are no $S$-type domain walls between the states $D_{11}$ and $D_{12}$ in the monoclinic phase, and only two possible $W$-type walls with fixed Miller indices correspond to planes (001) and (100) in the monoclinic setting. It should be noticed that the described case is a compound twin (Table III), when a twin individual can be considered as a reflection twin or an axial twin at the same time. The pairs of orientation states $D_{11}-D_{22}, D_{11}-D_{i 1}(i=2-6)$ in the monoclinic phase can be connected by $W$-type domain walls as well as by $S$-walls (Table III). In the first case, the states are reflection twins, in the second case they are axial twins.

Various four-domain configurations can appear in the low-symmetrical monoclinic phase as a result of numerous domain states $(q=12)$ and, correspondingly, allowed domain walls between those domains (Table III). In the monoclinic phase, only intersections of $W$ - and $S$-type walls are possible. These configurations can be divided in several groups [32]. In the sample volume the translation of such junctions forms a chevron-like pattern.

\section{Chevron-like domain structure in materials with martensitic phase transitions}

The formation of a chevron-like domain structure in a rectangular plate of single domain ferroelastics is sketched in Fig. 12. Due to twinning shear in the direction ${ }^{A} \eta_{1}$ (normal to the figure plane), of the monodomain plate (Fig. 12a), bidomain configuration (Fig. 12b) or lamella-like domain structure with alternating domain areas $M$ and $A$ can appear (Fig. 12c). Under another twinning shear along the direction ${ }^{B} \eta_{1}$ (parallel to the figure plane) sample can form two domains (Fig. 12d) or twin lamella $B$ can appear (Fig. 12e). In this case, the shift of some areas of the crystalline plate along direction ${ }^{A} \eta_{1}$ (parallel to walls of lamella $B$ ) results in the formation of lamella $A$ in the "matrix" part $M$, whereas lamella $C$ forms in lamella $B$ (Fig. 12f). A chevron-like ferroelastic domain structure results and the surface of the previously monodomain plate becomes corrugated. Such feature is favored in materials with martensitic phase transitions [38]. It is significant that the twinning shear along direction ${ }^{B} \eta_{1}$ in the sample with lamella $A$ (Fig. 12c) does not lead to a chevron-like domain structure (Fig. 12f). In the matrix part $(M)$, such twinning shear can result in the formation of $B$-type lamella, which will be blocked by already existing lamella $A$.

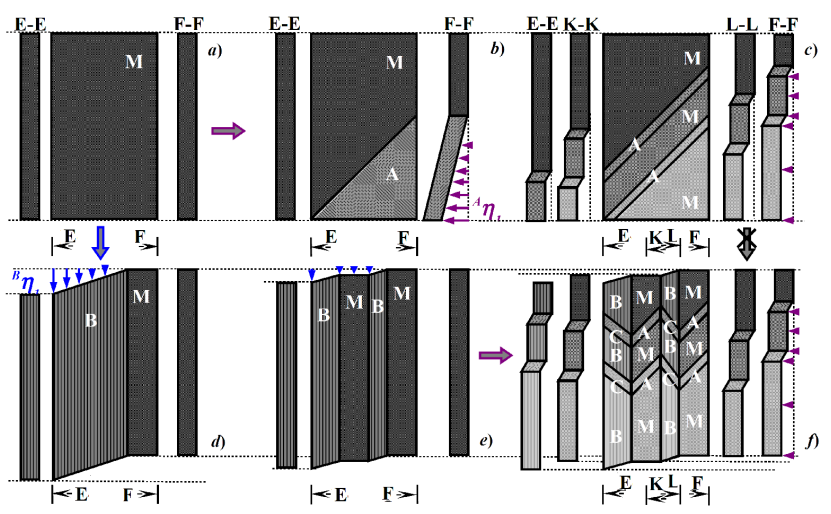

Fig. 12. Sketch of the formation of a chevron-like domain structure in a volume of rectangle plate of single domain ferroelastics.

\section{Conclusions}

The crystal structure of LSGM05 can be considered as a small deformation of an ideal cubic (paraelastic) perovskite cell with point group symmetry $G=m \overline{3} m$. The deformation produces the crystal structures of the point group symmetry $\overline{3} m$ and $3 m$ in the trigonal phase, the point group symmetry $2 / m$ in the monoclinic phase, and the point group symmetry $\mathrm{mmm}$ in the orthorhombic phase. There may be 4 orientation states in the centrosymmetrical trigonal phase, 8 domain states - in the non-centrosymmetrical phase, 12 in the monoclinic phase, and 6 in the orthorhombic one.

All possible domain pairs in the trigonal phase are ambivalent and can be separated by $W$-type walls with integer Miller indices. These domain walls are parallel to lateral and diagonal faces of the pseudo-perovskite cell. In terms of the mechanical twinning theory such bidomain configurations are classified as compound twins.

Such compound twins are the domain pair $D_{1}$ and $D_{2}$ with domain walls parallel to lateral faces (100) and (010) of the pseudo-perovskite cell in the orthorhombic phase of LSGM05. Between other domain pairs $D_{1}-D_{i}(i=3-6)$ domain walls of $W$-type with orientations (101), (101), (011) and (011) (diagonal faces of pseudo-perovskite cell) 
can appear as well as $S$-type domain walls, which propagate via lateral diagonals of the pseudo-perovskite cell.

During the phase transition from orthorhombic to monoclinic phase each of the 6 orientation states can be divided into two domain states. At the same time all possible domain walls, which are allowed in the orthorhombic phase, can also appear in the monoclinic phase. Apart from the abovementioned walls in the monoclinic phase of LSGM05 $W$-type walls can form with orientation (100) and (001) and $S$-type, which propagate via pseudo-perovskite axes [100] and [010]. It should be noted that some domain states cannot be adjacent.

In all three phases the twin structure tends to form a "chevron-like" wall configuration that allows for the stress-free coexistence of four different orientation states in LSGM05. Such patterns of domain walls are expected to be characteristic also for other perovskite-type compounds with a sequence of ferroelastic phase transitions related to LSGM05. Examples are mixed conductivity perovskites, which are used as electrodes and interconnectors in SOFC batteries.

\section{Acknowledgments}

The work was supported by WTZ (UKR 07/009) and Ukrainian Ministry of Education and Science (the "Tern" project).

\section{References}

[1] A.B. Stambouli, E. Traversa, Renewable Sustainable Energy Rev. 6, 433 (2002).

[2] J. Huijsmans, F. Berkel, G. Christie, J. Power Sources 71, 107 (1998).

[3] N. Sammes, R. Boersma, J. Power Sources 86, 98 (2000).

[4] B.C.H. Steele, A. Heinzel, Nature 414, 345 (2001).

[5] S. Haile, Materials Today 6, 24 (2003).

[6] N.Q. Minh, J. Am. Ceram. Soc. 76, 563 (1993).

[7] M. Feng, J.B. Goodenoough, Eur. J. Solid State Inorg. Chem. 31, 663 (1994).

[8] T. Ishihara, H. Matsuda, Y. Takita, J. Am. Chem. Soc. 116, 3801 (1994).

[9] S.J. Skinner, J.A. Kilner, Materials Today 6, 30 (2003).

[10] K. Huang, J. Wang, J.B. Goodenough, J. Mater. Sci. 36, 1093 (2001).

[11] A. Aird, M.C. Domeneghetti, F. Mazzi, V. Tazzoli, E.K.H. Salje, J. Phys., Condens. Matter 10, 569 (1998).
[12] W.T. Lee, E.K.H. Salje, U. Bismayer, Phase Transit. 76, 81 (2003).

[13] M. Bartels, V. Hagen, M. Burianek, M. Getzlaff, U. Bismayer, R. Wiesendanger, J. Phys., Condens. Matter 15, 957 (2003).

[14] S. Stemmer, A. Sane, N.D. Browning, T.J. Mazanec, Solid State Ionics 130, 71 (2000).

[15] C.L. Jia, Science 303, 2001 (2004).

[16] M. Kurumada, E. Iguchi, D. Savytskii, J. Appl. Phys. 100, 014107 (2006).

[17] E. Iguchi, D. Savytskii, M. Kurumada, in: Diffusion and Reactivity of Solids, Ed. Ja.Y. Murdoch, Nova Sci. Publ., New York 2007, p. 115.

[18] G. VanTendeloo, D. Broddin, H.W. Zandbergen, S. Amelinckx, Physica C 167, 627 (1990).

[19] A. Putnis, E.K.H. Salje, Phase Transit. 48, 85 (1994).

[20] Yi. Zhu, M. Suenaga, A.R. Moodenbaugh, Philos. Mag. Lett. 62, 51 (1990).

[21] R.F. Klie, Y. Ito, S. Stemmer, Ultramicroscopy $\mathbf{8 6}$, 289 (2001).

[22] F. Tsai, V. Khiznichenko, J.M. Cowley, Ultramicroscopy 45, 55 (1992).

[23] Y. Wang, F. Guyot, R.C. Liebermann, J. Geophys. Res. B 97, 12327 (1992).

[24] J. Fousek, V. Janovec, J. Appl. Phys. 40, 135 (1969).

[25] J. Sapriel, Phys. Rev. B 12, 5128 (1975).

[26] V. Janovec, Czech. J. Phys. B 22, 974 (1972).

[27] O. Mügge, Neues Jahrb. Mineral. Geol. 98 (1889).

[28] G. Friedel, Leçons de cristallographie, Berger-Levrault, Paris 1926.

[29] R.W. Cahn, Adv. Phys. 3, 363 (1954).

[30] R.W. Cahn, Acta Metallurgica 1, 49 (1953).

[31] M.V. Klassen-Nekludova, Mechanical Twinning, Nauka, Moskva 1960 (in Russian).

[32] D. Savytskii, U. Bismayer, Phase Transit. 81, 431 (2008).

[33] K. Aizu, Phys. Rev. B 28, 754 (1970).

[34] K. Aizu, J. Phys. Soc. Jpn. 28, 706 (1970).

[35] A.L. Roytburd, Phase Transit. 45, 1 (1993).

[36] V.K. Wadhawan, Phase Transit. 3, 3 (1982).

[37] L. Vasylechko, V. Vashook, D. Savytskii, A. Senyshyn, R. Niewa, M. Knapp, H. Ullmann, M. Berkowski, A. Matkovskii, U. Bismayer, J. Solid State Chem. 172, 396 (2003).

[38] A.L. Roytburd, Izv. AN SSSR, Ser. Fiz. 47, 450 (1983) (in Russian). 